\title{
The correct development of primary object relationships the oral personality
}

\author{
Maria Grazia Spurio ${ }^{1,2 *}$ \\ ${ }^{1}$ Psychotherapist, Master in Neuropsychiatry and Forensic Psychology, Italy \\ ${ }^{2}$ Director of Genius Strategic Counselling School via C.A. Jemolo 83 ROME, Italy
}

\begin{abstract}
The care of the primary relationship is the most important and necessary prevention of a society, and every relationship, even the most important and intimate, has unfortunately become secondary to the demands of productivity, economy, consumerism, compared to an amorphous and dominant collective. Taking care of the child 's original relationship, al-lows to do primary prevention, which means to radically and scientifically prevent future disturbances of personality, of behaviour and development of children and future adults. The pathologies that affect the oral cavity are extremely widespread: one can mention, for example, the very high incidence of odontostomatological affections as well as other pathologies, serious, to have an idea of how much this problem is major. For this reason, it is evident the importance that psychotherapy can play with respect to the support and to the help given to an individual in the process of interpretation of life situation.
\end{abstract}

\section{Introduction}

If we accept the assumption that each pathology, in a certain sense mirrors the social organization of the civilization, the consequence is that the vertiginous increase of "border-line pathologies" of the last decades reflects the modifications in the social structure that seem to have the ability to undermine the proper development of "primary object relation-ships".

Problems mainly arise when the traditional educational methods of a society become gradually less capable of preparing children to face the requests they will meet once adults. This is partly due to of a form of disinterest, or social disengagement, towards the early stages of life and on their impact on the society that new generations will set up. That's why, but not the only reason, it is important to say that no culture can foresee so well the future to prepare each of its components to live it without problems.

Moreover, each society, because of its order, can better provide aid for some crises more than for others. For these reasons it would be desirable for psychotherapists, families, educators and scholars in general of the field of peer education and child-hood, that they could optimize and co-ordinate efforts and interventions, in order to contribute to the development of a society more capable of preparing its children for the role and the difficulties they may encounter once they become adults.

As a first research area, one might begin to investigate, for example, how inadequate development during growth affects adult behaviour. In other words, each step of psychosocial development, has a goal that children must reach, so, what will be the consequences of adult behaviour in case of failure in achieving goals in the period of growth?

Freud (1922\&1925) [1,2] described the functioning of psychic evolution through the various phases that characterize the psychosexual development, from the moment that the desired object is invested by the limbic energy, other authors, on the other hand, were more focused on to the earlier phases, the period in which the Subject
'Self' is not yet a mentally differentiated structure from the other object, so, the object of the impulsive investment is the symbiotic union of the 'Self' and of the 'Other', in what was called the phase of primary and omnipotent narcissism.

This phase of psychic development has attracted the interest of many post-Freudian psychoanalysts, beginning with the pioneering studies of Melanine Klein (1932) [3] and Margaret Mahler (1975),[4] and then with a great number of researches whose common denominator may be traced back to the importance which is conceptually attributed to the primary bond, "pre-sexualized", that the infant establishes in the early months of his/her life with his/her mother. Giving most of the attention to the mother / baby relationship is fundamental in these stages of pregnancy and in the first year of life, this determines the child's future personality. It is during this period that the basic foundations of the 'house' of personality are built, on which all the other plans will be based. The experience of this phase, there-fore, will positively or negatively affect the future stages and growth crises.

\section{Primary prevention in the neonatal phase and psycho- logical disorders in the adult personality}

Birth, as well as death, is a sacred moment, consequently, according with many re-searchers and thinkers, it is necessary that one gives the best care and support possible, in fact this is exactly what characterizes the civilization of a population. The care of the primary relationship is the most important and necessary prevention of a society, and every

${ }^{*}$ Correspondence to: Maria Grazia Spurio, Psychotherapist, Master in Neuropsychiatry and Forensic Psychology Director of Genius Strategic Counselling Schoolvia C.A. Jemolo 83 ROME, Italy, Tel: 92-00187377-7; E-mail: dott.m.g.spurio@ hotmail.it

Key words: orality, child, development, adult, pathology

Received: January 10, 2018; Accepted: February 12, 2018; Published: February 16,2018 
relationship, even the most important and intimate, has unfortunately become secondary to the demands of productivity, economy, consumerism, compared to an amorphous and dominant collective.

It is therefore fundamental, for our society, to regain the sense of individuality, uniqueness and originality, and all this can only be restored by the care and respect for the beginning of life. In other words, it is only paying attention to this dual union of the first phase of life, in which the identity and the uniqueness of the individual begins to flourish, that it is possible to promote the healthy development of the personality. It is therefore necessary to give to this period the most of one's attention, because it is an extremely delicate and fragile moment, an unrepeatable time for the child, since every negative or positive experience, even the smallest one, causes strong emotions, fears and anxieties that will impact exponentially on the development of his/her personality and the successive experiences of life.

Consequently, the mother, and her ability to understand and positively welcome the child's experiences, has a fundamental role in this process, and she will decisively determine the future integrative and elaborative skills of the child. This "first bond" or "primary object relationship is a total, unconditional need for absolute love. It is therefore of utmost importance that both the mother and the child are assisted and supported in knowing and man-aging this period of life from a social and cultural point of view. Healthcare professionals, specifically prepared on these phases and on family support, are extremely important. They must be capable of recognizing newborn signals and symptoms, and able to relate them to life experiences and to contain parents' anxieties.

Taking care of the child 's original relationship, allows to do primary prevention, which means to radically and scientifically prevent future disturbances of personality, of behaviour and development of children and future adults. For example, it is evident the importance of allowing mothers to take maternity leave until the end of their first year of life to be close to their child; that they should be protected as much as possible with kindergartens in the workplace or at home to help the child not to lose its familiar context

A specific discussion must be made for hospitals. To prevent any type of illness, it is fundamental to take care both of the mother and the child during the neonatal phase. One cannot consider a newly born baby as any other adult patient, with his room, his assistant washing and changing, with the only difference that, for feeding, it is necessary the mother's presence.

Since the infant is an indivisible unit with the mother, she can neither be separated from him/ her even for few hours nor be replaced by unrelated ones. The absence of the mother determines significant anguish that child cannot face alone, and that remain even if the child stops crying and seems to settle down.

Even more serious is the baby's isolation due to premature childbirth or a serious disease. It would be necessary, also in this case, to allow the mother to stay in the hospital together with the child, because she gives $\mathrm{him} /$ her crucial energy and strength to face the disease, as she is the source of love and life for her child. The lack or the shortage of this constitutive relation of the human psyche, which lay the foundations of subsequent objection relations and thus social, has been analyzed by different authors from various points of view. A great number of experts, we can mention for example Balint (1991), [5] Bowlby (1988) [6], and Winnicott (1995), [7] agree that the ungratified need of this kind of "primary love" causes serious distortions in the development of the ego, and more generally of the psychic organization.
This deficiency is the basis of ego pathologies, classified as "borderline personality disorders", whose main feature is an insecure "attachment" to the desired object, characterized by a fundamental and unresolved ambivalence: on the one hand, a need of love, extreme because unsatisfied, on the other hand, a blind and destructive hate. The lack of a stable emotional "center of gravity" leads these individuals to create fragile and inconstant emotional relationships, more dominated by the need to repair the original narcissism "injury" (see, for example, Schellenbaum, 1991 [8]), than by a mature sexual relationship where the libido is free to address to the desired object of love.

\section{Oral personality}

In the Freudian conception (1922), "orality" is defined as a stage, that is, a phase of the development of the individual, and more precisely the first of the three phases who's the successive are the anal and the genital ones. According with the principles of W. Reich's theories, the issues related to the mouth and to some personality disorders in the adult life, are determined by 'fixation' to the oral phase. Professor Federico Navarro, psychiatrist and psychotherapist, founder of the Reichian movement in Italy, said, “... being the mouth in function of orality, all the diseases related to it, have to do with orality ...”.

In fact, the pathologies that affect the oral cavity are extremely widespread: one can mention, for example, the very high incidence of odontostomatological affections as well as other pathologies, serious, to have an idea of how much this problem is major. There is, among other things, another consideration to be done: it seems that most people with psychological disturbances have issues related to 'fixation' at the oral stage, and although this could not be predominant, some traces can be found in almost all subjects who are in psychotherapy treatment. The oral phase is the first stage of psycho-sexual development and focuses on the mouth.

It is known that children try to put everything in their mouths, which is the cognitive organ for excellence. Through this mucous membrane, the child enters in connection with the mother and through her, it is possible to taste the pleasure of exploring the external world. Oral fixation represents a fixation at this stage, and it retains some of its features. Oral fix-ations have, in common, the feature to tend to behaviours which involve the oral cavity: eating, smoking, drinking, sucking, etc. The oral personality is greedy to take from the outside world and to hold back its elements. These people have a marked narcissistic tendency, focusing on themselves; they do not recognize the others as separate bodies from them-selves, as they are considered only in relation to what they can offer, as if they were a nourishment.

Behavioural manifestations of this type of personality in adults include: smoking, alcoholism, but also logorrhoea. Oral type always asks something, through an attitude sometimes aggressive and sometimes timid and imploring. From a behavioural point of view, the individual might manifest an inclination to victimise, to regress to a state of dependence and / or to develop orally dependent practices such as those described, or manifesting a strong dependence on food, or even constituting a sarcastic or pungent personality. We can define 'regression' the unconscious return to objects and methods of gratification belonging to earlier stages of libidinal development and / or previous object or identification relation-ships, and it indicates a defensive mechanism. This attitude tends to emerge when the individual faces highly conflicting situations: the return to behaviours that belong to past periods of life, considered less complex by the person, is more rewarding than the present. A reason that should be considered to 
argue the enormous spread of this problem is, in my opinion, largely attributed to the bad relationship the child had with his mother. It is known that the mouth, for a new born baby, is the first organ through which one enters sensory and cognitive contact with the outside world.

The mouth, in addition to the role of intermediary for these contacts, fulfils an equally important function, that of sucking and ingesting food. In this case, much more significant than the quantity, is the quality of the child - parent relationship. Through sucking, the baby does not only satisfy the feeding needs but establishes that bond with the moth-er, made of love, security and tenderness. Unfortunately, in some cases, this type of relationship is not easily established because of possible disorders related to the maternal psychic sphere, so the child does not receive adequate care or the safety and affection he/ she needs. In addition to the problems caused by a bad mother-child relationship, one must keep in mind the tremendous psychological conditioning that the present society has on the individual. This imagebased, extremely competitive society, finally creates personalities with distinct narcissistic tendencies. Since, however, the narcissistic position is al-most always a cover, it happens that the subject, after having faced a frustration on the side of competitiveness, regresses into an oral position, with, sometimes, depressive aspects, that an expert professional is able to catch [9].

This shows that one of the most common defence mechanisms is the oral regression. An individual usually responds to frustration with an oral regression, for example looking for food to find the satisfaction otherwise denied by external events. In fact, it is com-mon to find depressive type among the obese people. However, it is important to remember that what people manifest in adulthood, with regards to the psychic sphere, besides being the result of contingent factors, sinks the roots in the one's personal history of life. This means that our behaviour in adulthood is strongly conditioned by our personal experience with the emotionally significant figures of our childhood and, above all, as already mentioned, with the maternal figure. The regression to an oral position, leads the subject to reactivate those typical requests of the child that originally involved the oral sphere, as well as the affective ones, in relation to food. The imprinting, the memory, however, are linked to a satisfaction of material type, the food ones. Many mothers still react to the restlessness and to their children's' cries through feeding them, so they are systematically gorged with food at the slightest complaint. Young children, unfortunately, do not speak and express themselves with tears: who knows if, instead of food, it would better take them in arm, cosset them with love? After all, their primary need is precisely to be loved.

\section{Imagination as a means of control}

In the Erikson's theory the central idea is that in every culture, the experts who deal with paediatrics, psychology and educational development, should be, more than ever, aware of psychosocial conflicts which typify a culture, and consequently they should pro-mote and deal with those development paths that have good possibilities to solve those specific difficulties. Like Freud, Erikson also believed that problems arising in early childhood can last for a lifetime. For example, the suspicious and pessimistic adult, or who continually doubts his abilities, has probably been a child who has not developed sufficient self-trust and has failed to obtain sufficient autonomy. Still, according to Erikson, some destructive and paranoid attitudes that can be found in the adult who defines an-other one as different or negative person, go back to the second phase of development, in which the child faces the conflict between autonomy and shame and doubt. Furthermore, the degeneration to which this phase can lead is called by Erikson "legalism": satisfaction in punishing rather than in understanding and compassion.

The consequences and the strongly negative impact that this type of personality can have on the social and personal fabric of the adult personality remain evident. Erikson has also developed a more specific mechanism of development as a system of facing difficulties, the game. In this context the term game is to indicate the use of imagination to try ways of mastering and adapting to the world, to express emotions, to search for past situations or imagine future ones, and to develop new models of existence. The game, therefore, is not limited to the child, but it is always present in later times, for example in the adolescent, who, with his/her imagination, starts to be interested in various things, as well as and in the adult. The game is often ritualized and becomes normal in a certain way, a culturally accepted way of interacting with others.

In addition to the game to create new ways of developing imagination and new models of existence, another tool that could expertly be integrated into the psychotherapeutic path, is guided fantasies. Mental stimulation achieved through regressive techniques and guided fantasies, manages to create right synaptic connections, in order to focus the thought on specific inner images adapted to the situation of the individual and, consequently, generate the appropriate positive emotions to bring back energy and serenity, this all with the precise objective of freeing the molecules that increase well-being and the level of excellence of the immune system. Some psychotherapeutic techniques such as deep relaxation techniques, guided fantasies, regressive therapies, hypnosis, do not dull the attention but enhance it, going to solicit brain areas different from those related to the sphere of rationality and they also allow greater control of neuroendocrine circuits, particularly that of stress, as well help to have a greater brain coherence, better communication between the two hemispheres and greater adaptability (Bottaccioli, 2005).

Everyone, during his life, from the first moment of life to the last one subjected to a series of stimuli. But it is the meaning that one will attribute to each of them that will determine the nature of the activated responses, both in terms of entity and duration. These answers will be revealed functional or dysfunctional. (Spurio) Behind ideas and thoughts, there is a chemical connection with our biological system. A key role in this process is the hypothalamus, because of his function in hormonal regulation. The peculiarity of this small structure is that it is particularly sensitive and reactive to the thoughts, memories, interpretations of present stimuli or anticipations of future events. Therefore, in addition to how is constituted the bio psycho social environment in which an individual spends his life, the fundamental question is how the person interprets and perceives the reference environment. This is because it is right this "interpretation" that gives the go-ahead to the consequent bio chemical responses, for example when the nervous system is stimulated to evaluate a positive situation, it will start producing dopamine, serotonin, oxytocin etc., a biochemical mixture that involves the whole organism with undoubted benefits for the individual.

On the contrary, if we rate a situation as frightening, or we must face a frenetic and anxious life, or, still, if the individual spends a significant part of his/her time in toxic social relationships, his/her body will consequently not activate a bio-chemical response favourable to health and well-being. It can therefore be affirmed that it is not only the reality that determines the type of bio-activated chemical response, but the 
perception and interpretation of it, given by the individual oneself. For this reason, it is evident the importance that psychotherapy can play with respect to the support and to the help given to an individual in the process of interpretation of life situation.

During a psychotherapeutic support path, the use of imaginative modalities like games or guided fantasies as an additional tool, in comparison with traditional therapy, can be very helpful to face, remodelate and stimulate different brain areas compared to those related to the sphere of rationality, and it also allows a greater control of neuroendocrine circuits.

\section{References}

1. Freud S (1922) The Ego and the Ego (Italian edition) in Opere, vol.9, Boringhieri, Torino.
2. Freud S (1925) Inibizione, sintomo e angoscia,(trad. it.), in Opere, vol.10, Boringhieri, Torino Freud A., L'Io e i meccanismi di difesa (trad. it), in Opere, vol.1, Boringhieri, Torino

3. Klein M (1932) The Psychoanalysis of Children, (trad.it.) Martinelli, Florence.

4. Mahler M, Pine F, e Bergman A, (1975) The psychological birth of the child, (trad.it.), Boringhieri, Turina.

5. Balint M (1991) The primary love, Raffaello Cortina Editore, Milan.

6. Bowlby J (1988) Attaccamento e perdita, Bollati-Boringhieri, Torino.

7. Winnicott D (1995) Affective development and environment. Studies on the theory of affective development, Armando Edito-re, Rome.

8. Schellenbaum P (1991) The wound of the unloved, RED Edizioni, Rome.

9. Hartmann, Kris e Lowenstein (1946) Comments on the formation of psychic structure, The Psychoanalytic Study of the Child 2:11-38.

Copyright: $@ 2018$ Spurio MG. This is an open-access article distributed under the terms of the Creative Commons Attribution License, which permits unrestricted use, distribution, and reproduction in any medium, provided the original author and source are credited. 